\title{
Depression im Alter: Ist Sport wirklich eine effektive Therapie?
}

Depressionen stellen die häufigste psychische Erkrankung im höheren Lebensalter dar und sind mit erhöhter körperlicher Morbidität und Mortalität verbunden. Sport und Bewegung sollen helfen. Wie effektiv diese Maßnahmen sind, wurde jetzt in einer Metaanalyse untersucht.

— Für die Metaanalyse konnten sieben Studien ausgewertet werden. In der Regel wurde ein gemischtes Training durchgeführt, das sowohl Kraft- als auch Ausdauerkomponenten enthielt. Typischerweise gab es drei bis fünf Trainingseinheiten pro Woche (30-45 Minuten) über einen Zeitraum von drei bis vier Monaten.

Es ergab sich eine Assoziation zwischen sporttherapeutischer Intervention und signifikant niedrigeren Depressionswerten. Dies war unabhängig davon, ob die Diagnose in den Studien klinisch oder über eine Symptom-Checkliste gestellt wurde. Die Ergebnisse zeigen, dass auch bei älteren Menschen mit klinisch relevanten depressiven Symptomen ein den individuellen Fähigkeiten angepasstes therapeutisches Sportprogramm zu einer Abnahme der depressiven Symptomatik führt.

\section{- Bridle C, Spanjers K, Patel S et al.}

Effect of exercise on depression severity in older people: systematic review and meta-analysis of randomised controlled trials. Brit J Psychiatry 2012; 201: 180-185

\section{Kommentar}

Die berechnete Effektstärke der sporttherapeutischen Intervention (standardized mean difference $=S M D=0,34$, Konfidenzintervall 0,17-0,52) liegt in derselben Größenordnung wie die Effektstärken für antidepressiv wirkende Medikamente (SMD =0,2-0,5) oder psychotherapeutische Verfahren (SMD =0,18-0,34). Die große klinischtherapeutische Herausforderung liegt darin, für den individuellen Patienten mit einer depressiven Erkrankung ein tägliches Bewegungsprogramm zu finden und umzusetzen, das die sehr unterschiedlichen körperlichen Beeinträchtigungen dieser Patientengruppe berücksichtigt.

A. BROOCKS =

Sport könnte ihm helfen. 